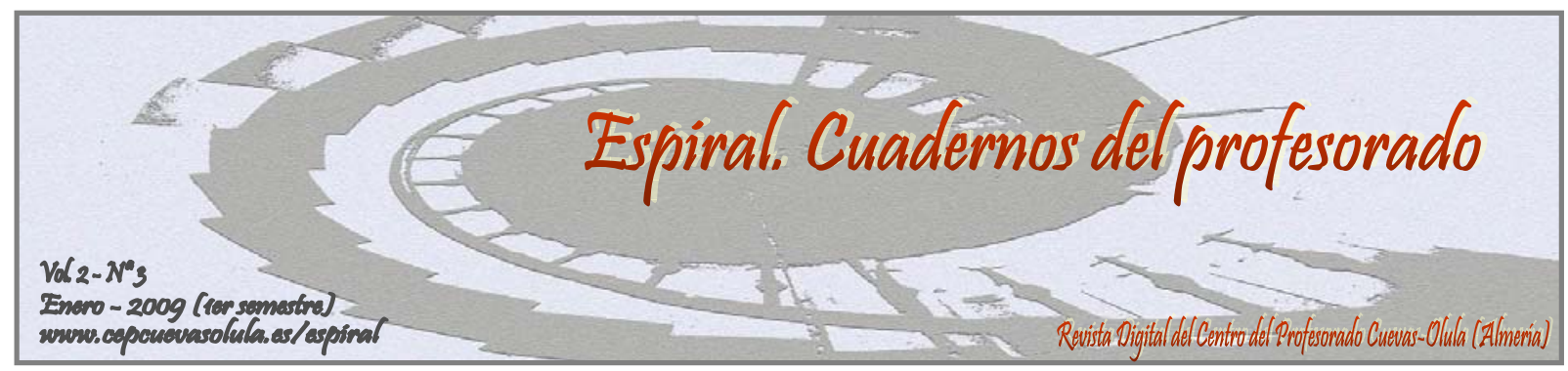

\title{
ANÁLISIS DE LAS ACTIVIDADES REALIZADAS EN LOS PROYECTOS DE INTERCULTURALIDAD DESARROLLADOS EN LAS PROVINCIAS DE ALMERÍA Y MÁLAGA
}

\author{
ANALYSIS OF ACTIVITIES IN PROJECTS IN DEVELOPED \\ NTERCULTURALITY ALMERIA AND MÁLAGA
}

\author{
María Fátima Cobo Castillo ${ }^{(1)}$, María Angustias Motos Martínez ${ }^{(1)}$, Antonio José \\ García Girona $^{(1)}$ y Gabriela Moral Torralbo ${ }^{(2)}$ \\ ${ }^{(1)}$ Equipo de Orientación Educativa de Cuevas del Almanzora, Almería (España) \\ ${ }^{(2)}$ Centro del Profesorado Cuevas-Olula, Cuevas del Almanzora, Almería (España)
}

\begin{abstract}
RESUMEN: Uno de los fenómenos más relevantes en el actual panorama educativo radica en la presencia cada vez más creciente de alumnado procedente de una diversidad étnica, lingüística y cultural desconocida anteriormente en nuestro sistema educativo y por tanto el profesorado se encuentra en estos momentos con el reto de construir aulas y escuelas interculturales como camino de construcción de una ciudadanía intercultural que asuma la diversidad como algo positivo y enriquecedor para la propia convivencia social y educativa. Una de las principales herramientas comunitarias de carácter formativo del profesorado en sus propios contextos escolares es el proyecto de interculturalidad porque el profesorado puede acceder al conocimiento intercultural a través de la investigación y el aprendizaje compartido entre los diferentes miembros de la comunidad educativa. A lo largo de nuestra comunicación repasaremos como es recogida la educación intercultural desde el marco legislativo; estudiaremos los aspectos que se valoran positivamente en la elaboración de los proyectos interculturales desde la Dirección General de Innovación Educativa y Formación del Profesorado, y los contenidos y actividades que se llevan a cabo en centros educativos con proyectos de interculturalidad.
\end{abstract}

Palabras clave: interculturalidad, proyecto educativo, formación y actividades docentes.

ABSTRACT: One of the most social outstanding phenomenons in the current educational overview is the more and more presence of students from an ethnic, linguistic and cultural diversity before unknown in our educational system. Due to this situation, nowadays teachers are facing the challenge of building intercultural classrooms and schools as a way of creating an intercultural citizenship able to assume diversity as positive and enriching for the social and educational coexistence. One of the main community tools with training character for teachers within their school context is the interculturality project. This project is aimed to teachers in order to access to the intercultural knowledge through the shared investigation and learning between the different members of the educational community. Throughout our communication we will revise how the intercultural education is selected from the legal framework; we will study positive assessed aspects in the elaboration of intercultural projects from the General Directorate for Educational Innovation and Teacher Training, and the contents and activities carried out in educational centres in two Andalusian provinces (Almeria and Malaga) whose interculturality projects have been approved.Finally, our aim is to verify that the interculturality is being constructed as accurate activities are proposed and made within the school centres. In that way, the own teachers will have assumed and familiarized practically the idea at the end of the process.

Key words: interculturality, Educative Project, Educational Formation and Activities 
Cobo Castillo, M. F., Motos Martínez, M. A., García Girona, A. J. \& Moral Torralbo, G. (2009). Análisis de las actividades realizadas en los proyectos de interculturalidad desarrollados en las provincias de Almería y Málaga [en línea]. Espiral. Cuadernos del Profesorado, 2(3), 104-110. Disponible en: http://www.cepcuevasolula.es/espiral.

Enviar correspondencia a: GATA3121@terra.es

1.- LA INTERCULTURALIDAD EN EL MARCO LEGISLATIVO DE ANDALUCÍA.

La Comunidad Autónoma de Andalucía en el marco de las competencias que tiene transferidas en materia de educación ha elaborado una serie de planes y normas que nos ayudarán a entender el tratamiento a la interculturalidad.

La Ley 9/1999, de 18 de noviembre, de Solidaridad en la Educación tiene como objetivo, en su artículo 2.2., la potenciación del valor de la interculturalidad, integrando en el hecho educativo la riqueza que supone el conocimiento y respeto por la cultura propia de los grupos minoritarios. Parte de la población destinataria de esta Ley es la que pertenece a minorías étnicas, culturales o inmigrantes y las medidas que deben desarrollar los centros son las de favorecer el respeto de la identidad cultural, fomentar la convivencia y facilitar la participación del alumnado en el entorno social. El valor de la interculturalidad se favorece corrigiendo las actitudes de discriminación o rechazo que pueden producirse en el seno de la comunidad educativa.

Asimismo, en Andalucía se han desarrollado planes y programas globales para la atención educativa del alumnado inmigrante porque se considera que el fenómeno intercultural debe ser abordado no sólo desde el ámbito educativo sino desde una visión integral del fenómeno migratorio. En esta línea, se han elaborado dos Planes para la Inmigración en Andalucía ${ }^{1}$ con una serie de objetivos específicos dentro del área de intervención socioeducativa. De estos objetivos, nosotros nos hemos centrado en el 1.3. del II Plan Integral para la Inmigración de Andalucía 2006/09- con el que se pretende favorecer que los centros educativos elaboren proyectos de centro, que contemplen la perspectiva

\footnotetext{
1 I Plan Integral para la Inmigración en Andalucía 2001-2004, aprobado por el Decreto 1/2002, de 9 de enero. II Plan Integral para la Inmigración en Andalucía 2006-2009, aprobado por el Decreto 92/2006, de 9 de mayo.
}

intercultural, que faciliten y promuevan procesos de intercambio, interacción y cooperación entre las culturas, [porque] la educación intercultural implica el reconocimiento de la diversidad de culturas como un fenómeno positivo y deseable para todos los centros.

El desarrollo de un modelo de educación intercultural se concibe como una mejora de las actividades contenidas en el Proyecto Educativo de Centro, para conseguir el objetivo de aunar la diversidad, la igualdad de oportunidades, la integración socio-afectiva y la ayuda para la resolución de conflictos. Las medidas que se proponen para conseguir este objetivo son:

- La formación y asesoramiento específico al profesorado de los centros que atienden al alumnado perteneciente a familias extranjeras e inmigrantes.

- La publicación de materiales de apoyo para los centros y para el profesorado.

- El desarrollo de plataformas de intercambio de buenas prácticas en el ámbito de la formación del profesorado y la interculturalidad.

- El apoyo específico a los centros que hayan realizado proyectos de centro interculturales y planes de acogida.

A consecuencia de estas medidas en los últimos cursos 2006/07 y 2007/08 se han impulsado y facilitado el desarrollo de proyectos de interculturalidad, haciendo hincapié en la formación del profesorado para comprender el fenómeno de la inmigración. ${ }^{2}$

Con posterioridad a la puesta en marcha del II Plan Integral para la Inmigración 2006-2009,

\footnotetext{
${ }^{2}$ Instrucciones de 14 de junio de la Dirección General de Innovación Educativa y Formación del Profesorado por las que se regula la convocatoria de proyectos de interculturalidad 2006-2007.

Instrucciones de 24 de junio de 2007, de la Dirección General de Innovación Educativa y Formación del Profesorado por las que se regula la convocatoria de proyectos de interculturalidad 2007-2008.
} 
se ha publicado la Ley 17/2007, de 10 de diciembre, de Educación de Andalucía (LEA) que tiene como principio del sistema educativo el reconocimiento del pluralismo y de la diversidad cultural existente en la sociedad actual, como factor de cohesión que puede contribuir al enriquecimiento personal, intelectual y emocional y a la inclusión social (artículo 4.g).

\section{2.- EL PROYECTO INTERCULTURAL.}

Los cambios que se están produciendo en la sociedad, en especial los referidos a la integración de diferentes etnias, culturas, nacionalidades, etc., son un verdadero reto para el profesorado y toda la comunidad escolar. Ante esta nueva realidad se pueden tomar diferentes posturas. Dependiendo del momento histórico y del país, se han ido sucediendo diferentes respuestas: desde el aislamiento de las minorías por la cultura dominante al asimilacionismo, pasando por la multiculturalidad y en último término la interculturalidad.

Hasta no hace mucho, era extraño que un alumno o alumna de otra etnia ${ }^{3}$ se escolarizara en nuestras aulas, de tal forma que este hecho se consideraba exótico y se tomaba como anécdota ya que no era considerado como algo problemático. El centro docente actuaba como otro de los mecanismos de socialización y se producía el fenómeno de aculturación por el que la cultura minoritaria era absorbida por la mayoritaria. Sin embargo, en la actualidad, la integración de alumnado de otras culturas es habitual y por ello no se puede producir de igual forma que antes. Además, en ocasiones, la incorporación de estos estudiantes viene asociada con ciertas dificultades, tales como las de la comunicación, el desfase escolar, la precariedad económica, etc. No suele suceder que ante un alumno o alumna de otra cultura, que domine en cierta medida la lengua de acogida y disponga de un currículum similar o superior al del resto de los alumnos y alumnas de su grupo, existan conflictos escolares. Desde el sistema educativo, la diversidad cultural es en sí misma un elemento enriquecedor, que los docentes debemos saber aprovechar.

Hasta fechas muy recientes, la multiculturalidad ha sido considerada como un problema por resolver. Sin embargo, se están adoptando nuevos enfoques totalmente distintos que nos permiten ponderar que la

\footnotetext{
${ }^{3}$ Excepto alumnado de la etnia gitana, por supuesto.
}

interculturalidad es un aspecto enriquecedor del currículum escolar. Ya que no debemos limitarnos a dar respuestas a situaciones circunstanciales y aprovechar la interculturalidad como un recurso que enmarque el currículum de nuestros centros. La educación intercultural adopta así un sentido de enriquecimiento mutuo y de intercomunicación social. Con esta nueva metodología no se pretende cambiar ni transformar una cultura que puede ser rica y valiosa. Es nuestra obligación la de aprender y comprender, conocer y valorar a los demás como personas, como miembros de nuestra sociedad, que a la vez es la suya, y que con su presencia nos enriquecen, independientemente de su origen, raza o nacionalidad. Sin un conocimiento mutuo es imposible valorar, apreciar y adoptar una postura ante sus costumbres y tradiciones.

El proyecto intercultural es una propuesta contextualizada, singular y flexible que potencia los procesos de análisis, reflexión y debate sobre aquellos aspectos de la vida del centro considerados relevantes y que facilitan la toma de decisiones para la mejora de la práctica educativa orientada a la mayor calidad del aprendizaje del alumnado, la producción de conocimiento educativo y la construcción de comunidades de aprendizaje y educación. De tal manera que se transforma en una herramienta fundamental para el desarrollo integral del alumnado, de ahí, que sea fundamental el trabajo preparatorio para la elaboración y desarrollo de dichos proyectos.

\section{3.- MÉTODO.}

Para realizar esta investigación hemos recabado información a través de entrevistas a personal docente, así como el análisis de los blogs ${ }^{4}$ de 25 proyectos interculturales de las provincias de Almería y Málaga, analizándose las 170 actividades que a lo largo del curso 2007 y hasta junio de 2008 se han llevado a cabo en centros de Infantil y Primaria (CEIP) e Institutos de Educación Secundaria Obligatoria (IES). En nuestro estudio hemos clasificado las actividades realizadas en los centros en función de los aspectos que se valoran positivamente para la

\footnotetext{
${ }^{4}$ Blogs de Proyectos interculturales de la provincia de Málaga (CEP de Marbella-Coín): http://plataforma.cep-

marbellacoin.org/moodle/course/view.php?id=50 (Consulta 06/09/2008). Blogs de Proyectos interculturales de la provincia de Almería (CEP de Almería): http://www.cepalmeria.org/blogintercultural/?cat=1 (Consulta 06/09/2008).
} 


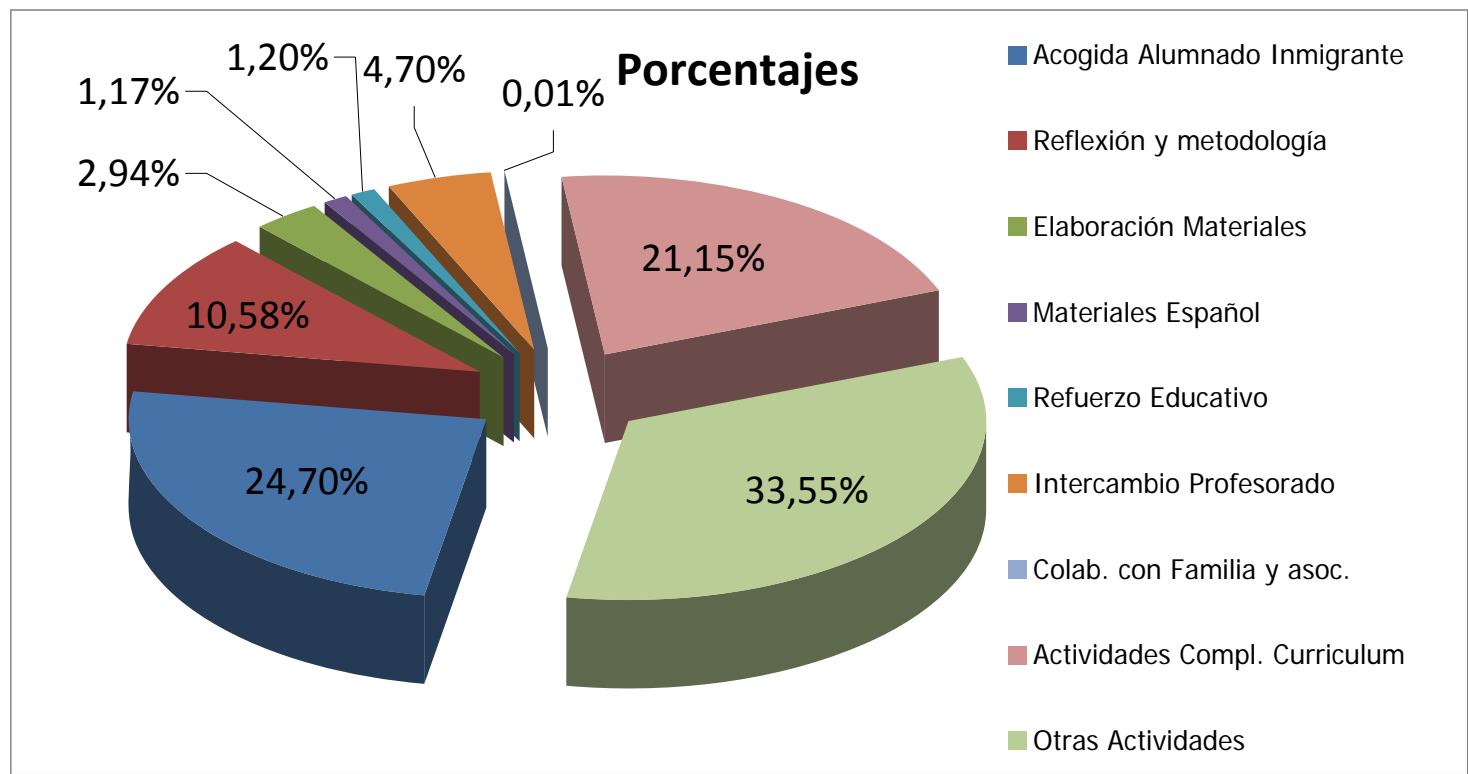

Figura 1.- Actividades desarrolladas según los criterios de valoración recogidos en las Instrucciones de 24 de junio de 2007 (porcentaje).

concesión de ayudas económicas con el objetivo de determinar el porcentaje de actuaciones de cada uno de ellos ${ }^{5}$. Concretamente se cita en dichas instrucciones:

Se valorará positivamente los proyectos que en su desarrollo atiendan a los siguientes aspectos:

- Procesos de acogida al alumnado inmigrante, estableciendo de manera expresa la organización del centro, la metodología, los materiales curriculares, las actividades, etc.

- La creación de cauces de reflexión sobre la práctica docente encaminada a encontrar metodologías, materiales, y dinámicas que permitan un trabajo en el que se atienda la diversidad cultural y lingüística de manera óptima.

- La elaboración, recopilación y utilización de materiales curriculares para la educación intercultural.

- La elaboración, recopilación y utilización de materiales para la enseñanza-

\footnotetext{
${ }^{5}$ Instrucciones de 24 de junio de 2007, de la Dirección General de Innovación Educativa y Formación del Profesorado por las que se regula la convocatoria de proyectos de interculturalidad 2007-2008.
}

aprendizaje del español al alumnado extranjero.

- Refuerzo de la enseñanza ordinaria mediante actividades extraescolares (estudio dirigido, refuerzo de áreas instrumentales, formación de padres y madres...)

- Intercambios del profesorado con los países de origen del alumnado inmigrante.

- Colaboración del centro con las familias y con aquellas asociaciones o entidades del entorno que estén implicadas en la integración social y la potenciación educativa de este alumnado.

\section{4.- RESULTADOS.}

Atendiendo a los criterios expuestos, los resultados obtenidos son los que siguen:

a) Los procesos de acogida al alumnado inmigrante, estableciendo de manera expresa la organización del centro, la metodología, los materiales curriculares, las actividades, etc. con $24,70 \%$ en actividades como por ejemplo:

- Elaboración y formación docente del Plan de acogida.

- Creación de un Departamento para la traducción de documentos.

- Nombramientos de mediadores interculturales. 
- Decoración de puertas para que aludan a la función que tiene ese espacio.

- Poner carteles con los nombres de las clases en diferentes idiomas y dando la bienvenida al centro.

- Concurso Web para fomentar en los centros educativos el diálogo intercultural.

b) La creación de cauces de reflexión sobre la práctica docente encaminada a encontrar metodologías, materiales, y dinámicas que permitan un trabajo en el que se atienda la diversidad cultural y lingüística de manera óptima con 10,58\% en actividades como por ejemplo

- Formación en currículo integrado.

- Formación en dinámicas de grupo.

- Formación en educación en valores.

- Formación en adaptaciones curriculares.

- Formación en la elaboración de materiales.

- Evaluación para la organización de la clase con el objetivo de toma medidas de atención a la diversidad.

c) La elaboración, recopilación y utilización de materiales curriculares para la educación intercultural con $2,94 \%$ en actividades como por ejemplo:

- Recopilación de material didáctico para la biblioteca.
- Uso del aula TIC como recurso didáctico.

- Compra de libros de carácter intercultural para la biblioteca del centro.

- Visita a la biblioteca y lectura de poemas en la lengua del alumnado inmigrante.

d) La elaboración, recopilación y utilización de materiales para la enseñanza-aprendizaje del español al alumnado extranjero con 1,17 en actividades como por ejemplo:

- Utilización de materiales elaborados por los propios docentes para el aprendizaje del español.

- Elaboración de materiales para la evaluación del nivel de competencia lingüística y curricular del alumnado extranjero.

e) El refuerzo de la enseñanza ordinaria mediante actividades extraescolares (estudio dirigido, refuerzo de áreas instrumentales, formación de padres y madres) con 1,2 \% en actividades como:

- Implicación de la familia de alumnado inmigrante en el aprendizaje de los hijos con la colaboración de las AMPAS.

- Participación de las AMPAS con las familias de alumnado inmigrante en otras actividades extraescolares (como por ejemplo la creación de un taller de lengua materna).

Tabla 1.- Actividades complementarias del currículum (21.15\%)

Educación Artística/Educación Plástica y Visual

- Hacer un mapamundi donde aparecen niños de diferentes razas y lugares con sus trajes típicos y banderas.

- Concurso de carteles para celebrar la semana intercultural.

- Concurso de carteles de la navidad del mundo.

- Elaboración de un mural gigante y multilingüe

- Construir decorados para teatro. que diga todos por la paz.

\section{Educación Artística/Música}

- Bailes típicos y danzas del mundo.

- Músicas del mundo.

- Viajes musicales por el planeta.

\section{Lengua Castellana y Literatura}

- Lectura de cuentos.

- Certamen de poesía.

- Montaje de cuentos tradicionales de diferentes países.

\section{Lenguas Extranjeras}

- Realizar felicitaciones de navidad en distintos - Recetas de comida en diferentes idiomas. idiomas.

\section{Conocimiento del Medio/Ciencias Sociales}

- Talleres para viajar a otros países.

- Exposición de trabajos sobre el arte en el mundo.

- Elaboración de un mapamundi. 
- Clases de lectura y escritura en español para madres de alumnos/as extranjeros.

f) Intercambios del profesorado con los países de origen del alumnado inmigrante con 4,70\% en actividades como:

- Visita de los docentes a centros educativos de los países Francia, Finlandia, Inglaterra y Rumania.

g) Colaboración del centro con las familias y con aquellas asociaciones o entidades del entorno que estén implicadas en la integración social y la potenciación educativa de este alumnado con $0 \%$. (figura 1 )

Por otro lado, consideramos que el $54.71 \%$ de las 170 actividades realizadas no son clasificables según los criterios aludidos. Un 21.15\% de este porcentaje son actividades complementarias de áreas del currículum ordinario como Educación Artística/Educación Plástica y Visual, Música, Lengua Castellana y Literatura, Lenguas Extranjeras, Conocimiento del Medio/Ciencias Sociales, mientras que el $33.56 \%$ podemos considerarlas como "otras actividades". Este $54.71 \%$ se desarrolla en la Semana Intercultural del centro y en las celebraciones del Día de la Paz, Día de Europa, Día de San Valentín, Día de Andalucía, Día de la Constitución, Día Internacional Contra la Violencia de Género, Día de la Navidad, Día Mundial del Sida. Ejemplos de estas actividades son: (tabla 1 y 2 ).

Tabla 2.- Otras actividades (33.56\%)

\begin{tabular}{ll}
\hline Juegos del mundo. & Globoflexia. \\
Concurso de gastrono-- & Manualidades \\
mía. & Desfiles. \\
Salidas y fiestas de & Artesanía. \\
convivencia. & Tatuajes. \\
Tómbola de juguetes & Taller de graffiti. \\
de segunda mano. & Exposición de trabajos \\
Pintura de caras. & manuales. \\
Taller de Henna & \\
\hline
\end{tabular}

\section{5.- CONCLUSIONES.}

Más de la mitad de las actividades que se realizan en los centros para fomentar la interculturalidad no están dentro de lo que la Administración Educativa valora para conceder los proyectos interculturales y sin embargo se llevan a cabo actuaciones que tienen como objetivo compartir, conocer las costumbres de todas y todos con la finalidad de que el conocimiento de los otros es considerado como algo primordial para la convivencia y el entendimiento.

El fomento de la interculturalidad ha de contemplarse como un proceso asumido por toda la comunidad educativa con finalidades $\mathrm{y}$ actuaciones programadas recogidas en los diferentes instrumentos de planificación docente del Proyecto Educativo de Centro, integrada y desarrollada en las diferentes áreas y elementos que conforman el currículo priorizando objetivos, contenidos y actividades de aprendizaje prestando especial importancia a los contenidos más perdurables, universales y transferibles, en definitiva a los más enriquecedores para todas y todos.

Así frente a un tratamiento folclorista o puntual de las diferencias culturales en los distintos centros educativos -efemérides, semana cultural, intercultural,...- conviene oponer un tratamiento que sirva para favorecer la adaptación y el progreso de las alumnas y alumnos en el marco de la cultura de acogida superando prejuicios y contribuyendo de forma eficaz a la integración socio-afectiva del alumnado.

Por lo tanto, la educación intercultural ha de dirigirse a todas las alumnas y alumnos, sean de la cultura que sea, como un modelo educativo que propicie el enriquecimiento cultural a través del intercambio, el diálogo y la crítica constructiva para el desarrollo de una sociedad basada en el reconocimiento de la dignidad de todos los seres humano; debiendo dar una respuesta pedagógica a la exigencia actual de preparar a los futuros ciudadanos para desarrollarse en una sociedad prácticamente multicultural e idealmente intercultural.

Del análisis de los datos extraídos de las actividades realizadas en los centros educativos, podemos deducir que el proceso de formación docente es fundamental para así poder elaborar y llevar a cabo propuestas educativas que desarrollen una verdadera educación intercultural en los centros escolares.

\section{6.- REFERENCIAS BIBLIOGRÁFICAS.}

I Plan Integral para la Inmigración en Andalucía. Consejería de Gobernación de la Junta de Andalucía (BOJA $\mathrm{n}^{\circ} 7$, de 9 de febrero de 2002).

II Plan Integral para la Inmigración en Andalucía. Consejería de Gobernación de la Junta de 
Andalucía (BOJA n ${ }^{\circ}$ 105, de 2 de junio de 2006).

CIDE (2005). La atención al alumnado inmigrante en el sistema educativo en España. Madrid: MEC.

SITE Consulting (2005). Evaluación externa del I Plan Integral para la Inmigración en Andalucía 2001-2004. Sevilla: Consejería de Gobernación.
Ley 9/1999, de 18 de noviembre, de Solidaridad en la Educación. BOJA nº 140.

Ley 17/2007, de 10 de diciembre, de Educación de Andalucía. BOJA nº 252.

Instrucciones de 24 de junio de 2007, de la Dirección General de Innovación Educativa y Formación del Profesorado por las que se regula la convocatoria de proyectos de interculturalidad 2007-2008. 